\title{
Epigenetic profiling of human brain differential DNA methylation networks in schizophrenia
}

\author{
Sheng-An Lee ${ }^{1}$ and Kuo-Chuan Huang ${ }^{2,3^{*}}$ \\ From 15th International Conference On Bioinformatics (INCOB 2016) \\ Queenstown, Singapore. 21-23 September 2016
}

\begin{abstract}
Background: Epigenetics of schizophrenia provides important information on how the environmental factors affect the genetic architecture of the disease. DNA methylation plays a pivotal role in etiology for schizophrenia. Previous studies have focused mostly on the discovery of schizophrenia-associated SNPs or genetic variants. As postmortem brain samples became available, more and more recent studies surveyed transcriptomics of the diseases. In this study, we constructed protein-protein interaction (PPI) network using the disease associated SNP (or genetic variants), differentially expressed disease genes and differentially methylated disease genes (or promoters). By combining the different datasets and topological analyses of the PPI network, we established a more comprehensive understanding of the development and genetics of this devastating mental illness.
\end{abstract}

Results: We analyzed the previously published DNA methylation profiles of prefrontal cortex from 335 healthy controls and 191 schizophrenic patients. These datasets revealed 2014 CpGs identified as GWAS risk loci with the differential methylation profile in schizophrenia, and 1689 schizophrenic differential methylated genes (SDMGs) identified with predominant hypomethylation. These SDMGs, combined with the PPIs of these genes, were constructed into the schizophrenic differential methylation network (SDMN). On the SDMN, there are 10 hypermethylated SDMGs, including GNA13, CAPNS1, GABPB2, GIT2, LEFTY1, NDUFA10, MIOS, MPHOSPH6, PRDM14 and RFWD2. The hypermethylation to differential expression network (HyDEN) were constructed to determine how the hypermethylated promoters regulate gene expression. The enrichment analyses of biochemical pathways in HyDEN, including TNF alpha, PDGFRbeta signaling, TGF beta Receptor, VEGFR1 and VEGFR2 signaling, regulation of telomerase, hepatocyte growth factor receptor signaling, ErbB1 downstream signaling and mTOR signaling pathway, suggested that the malfunctioning of these pathways contribute to the symptoms of schizophrenia.

Conclusions: The epigenetic profiles of DNA differential methylation from schizophrenic brain samples were investigated to understand the regulatory roles of SDMGs. The SDMGs interplays with SCZCGs in a coordinated fashion in the disease mechanism of schizophrenia. The protein complexes and pathways involved in SDMN may be responsible for the etiology and potential treatment targets. The SDMG promoters are predominantly hypomethylated. Increasing methylation on these promoters is proposed as a novel therapeutic approach for schizophrenia.

\footnotetext{
* Correspondence: d94922009@ntu.edu.tw.

2Department of Psychiatry, Beitou Branch, Tri-Service General Hospital,

National Defense Medical Center, Taipei, Taiwan

${ }^{3}$ Department of Nursing, Ching Kuo Institute of Management and Health,

Keelung, Taiwan

Full list of author information is available at the end of the article
}

(c) The Author(s). 2016 Open Access This article is distributed under the terms of the Creative Commons Attribution 4.0 International License (http://creativecommons.org/licenses/by/4.0/), which permits unrestricted use, distribution, and reproduction in any medium, provided you give appropriate credit to the original author(s) and the source, provide a link to the Creative Commons license, and indicate if changes were made. The Creative Commons Public Domain Dedication waiver (http://creativecommons.org/publicdomain/zero/1.0/) applies to the data made available in this article, unless otherwise stated. 


\section{Background}

Schizophrenia is a complex mental illness, which is caused by the malfunctioning of many genes. There are multiple neurophysiological causes for its symptoms. Approximately 1008 schizophrenia-related genes have been identified by genetic studies such as GWAS and methods of systems biology, which provides a global view of genetics of the disease [1]. Besides, in the postmortem brain samples of schizophrenia patients, 4116 schizophrenia marker genes have been found to show different expression patterns [2]. Previous studies have mostly focused on the discovery of schizophreniaassociated SNPs, genetic variants and more recently, gene expression patterns. However, more and more studies started to investigate the differences in epigenetics of schizophrenia patients. Epigenetics can provide important information on how the environment effects the genetic structure of the disease [3]. By crossreferencing the disease-related SNPs, the differentially expressed genes and the epigenetic changes, we may reveal the insights of schizophrenia etiology.

Methylation of genomic DNA could mediate gene expression. Although there has not been any specific methylated gene patterns identified for schizophrenia, there are significant associations between promoter CG islands (CGIs) hypermethylation vs. the up-regulation of genes, and the hypomethylation vs. the down-regulation of genes [4]. CGIs have been suggested to suppress gene expression by blocking the promoters. There are significant differences between the overall and specific methylation levels of the different tissue samples of schizophrenia [5]. Recent analyses of the methylation arrays of postmortem brain samples indicated that the hypermethylation of RELN, hypermethylation and down-regulated transcription of SOX10, and hypomethylation of MB-COMT promoters may contribute to the development of schizophrenia [6]. Genes such as COMT and REELIN, as well as a few others in the dopaminergic, serotonergic and GABAergic pathways, have also shown differential methylation profiles in schizophrenia samples [7]. Differential methylation has been noted in specific schizophrenic candidate gene groups (e.g. RELN, BDNF, COMT, 5-HTT and glutamate receptor genes) [8]. Global hypomethylation has also been noted in schizophrenia patients in experiments with larger sample sizes [9].

There are also evidences of the environmental factors that altered the methylation of genomic DNA in schizophrenia. Lower LINE-1 methylation in peripheral blood leukocytes has been noted in first-episode schizophrenia patients with histories of childhood trauma [10]. Genome-wide DNA methylation profiling of peripheral leukocytes conducted with 24 drug-naïve schizophrenic patients and 23 healthy controls showed that methylated
CpG sites were in $78.5 \%$ of the promotor regions in peripheral blood leukocytes [11].

The methylation and maintenance of $\mathrm{CpG}$ sites is crucial for neuron cell differentiation for the development of synaptic plasticity, learning ability and memory. Promotor hypermethylation of candidate genes in neurons is associated with transcriptional down-regulation of the corresponding mRNAs in postmortem studies of schizophrenia [12]. Differences in epigenetic patterns may contribute to phenotypic variations and disease susceptibilities [13]. Previous studies were mostly done on mouse models or stem cell lines [14, 15]. Nonetheless, a vast amount of methylation arrays of postmortem human brains have been released recently [16, 17]. These latest advances may implicate the importance of methylation patterns in schizophrenic patients. Most studies of gene methylation of mental diseases focused on the differential methylation patterns of genes. Little has been done on the correlations of differentially methylated genes and the expression of target gene.

Recent researches of schizophrenia tried to combine the methylation profiles of susceptible genes with network biology analyses. There are many studies attempted to locate differentially expressed schizophrenic genes on protein-protein interaction (PPI) networks and related pathways [2, 18-21]. Differentially expressed disease genes from postmortem brain samples of schizophrenia provide an overview of the disease maker genes. The analyses of disease PPI networks, the underlying pathways and protein complexes may construct a backbone for developing potential treatment strategy of the disease. Topologically and functionally important genes on the PPI networks may be seen as potential drug targets [19].

This study used a network biology approach to analyzed previous published DNA methylation profiles of the prefrontal cortex of 335 healthy controls and 191 schizophrenic patients [16]. In this dataset, there included 2104 CpGs identified as GWAS risk loci with differential methylation profile in schizophrenia [16]. Analyses of DNA methylation identified potential biological processes that regulate gene expression and contribute to disease mechanisms. We constructed the differential methylation and expression networks to interactions of methylated genes. Therefore, large scale analyses for differential methylation of schizophrenic susceptible genes were conducted and integrated with the differential expression data of schizophrenic susceptible genes to build the methylation-to-expression genetic network. The network explored the epigenetic mechanism of schizophrenic methylation networks, differential methylation pathways, complexes and corresponding biological 
functions. The genetic, epigenetic and transcriptomic information was integrated to give a comprehensive overview of schizophrenia.

\section{Methods \\ Selection of significant genes with differential methylation}

We downloaded published DNA methylation profiles of prefrontal cortex of 335 healthy controls and 191 schizophrenic patients from http://www.nature.com/ neuro/journal/v19/n1/extref/nn.4181-S12.csv, with permission from Macmillan Publishers Ltd: Nature Neuroscience, Jaffe et al. [16], copyright 2016. The list compares differentially methylated CpGs of patients with schizophrenia to adult healthy controls. Methylated genes were identified in the brain samples from the NIMH Brain Tissue Collection at the National Institutes of Health, the Offices of the Chief Medical Examiners of Columbia and the Commonwealth of Virginia, Northern District. DNA methylation was assessed using the Illumina HumanMethylation450 (450 k) microarray, which measures CpG methylation across $>485,000$ probes covering $99 \%$ of Ref-Seq gene promoters.

Schizophrenia differential methylation analysis was made among 335 patients with schizophrenia diagnosed by DSM-IV and 191 controls. A Chi-squared test $(\mathrm{df}=1)$ was performed to determine difference methylation significance. Hyper- or hypomethylation genes were calculated by moderated t-statistic values from 2104 CpGs. 1689 schizophrenic differential methylation genes (SDMGs) were selected. There were 688 differential methylated promotors, in which there were 16 hypermethylation promotors and 672 hypomethylation promotors.

The differentially expressed genes were collected from different schizophrenic candidate genes (SCZCGs) of differential expression gene databases from Huang, et al. [18], Huang, et al. [2] and Wu, et al. [22]. The significant differentially expressed transcripts with over- or under-expressed genes in the brain samples were selected by comparing the schizophrenia and control samples with Student t-tests. The corresponding $t$-test calculated the $p$-value for each gene. The genes with a $p$-value $<0.05$ were defined as SCZCGs. There are 615 over-expression genes and 1010 under-expression genes in SCZCGs. By exclusion of missing official symbols, a total of 1538 differential expression genes were selected for analysis.

For obtaining potentially involved pathways in schizophrenia, the pathway enrichment analysis of the significant differentially expressed genes are prioritized and the significance of corresponding pathways is ranked by $p$-value with FDR less than 0.05 using fdrtool [23].
Differential genetic methylation network in schizophrenia The schizophrenic differential methylation network (SDMN) was constructed for the comprehensive view of methylation profile in schizophrenia. The SDMN was generated by query-query protein-protein interaction (QQPPI) [24] and genetic interactions of SDMGs was recorded in version 8 of the Pathway Commons Database [25]. Pathway Commons Database [25] which collects BIND [26], BioGRID [27], CTD [28], DIP [29], HPRD [30], HumanCyc [31], IntAct [32], KEGG [33], NetPath [34], PANTHER [35], PhosphoSitePlus [36], PID [37], Reactome [38], SMPDB [39], TRANSFAC [40], MiRTarBase [41], DrugBank [42], Recon 2 [43], and WikiPathways databases [44] contain 34,661 molecular pathways.

The regulatory interactions and potential pathways of genes were investigated for understanding the disease mechanism of schizophrenia. To explore the modulation and regulatory relationships between the schizophrenic hypermethylated promotors and the differential expression genes, we identified the hypermethylated promotors and the Level 1 PPIs of SCZCGs to constructe the hypermethylation to differential expression network (HyDEN).

\section{Exploration of methylation profile and potential pathways} The biological features of schizophrenic methylation profile were discussed in this study. With the modularity of clique, complex and enrichment analysis of pathway, the bioinformatics analyses were performed for (a) the understanding of methylation profile interacted with differential gene expression in schizophrenia, (b) the discovery of potential cliques, complexes and involving pathways from enrichment analysis of SDMN, and (c) the evaluation of shared disease markers for schizophrenia and other major psychiatric disorders from PsyGeNET [45].

The clique and complex analysis was performed with data of the CORUM database [46] which has a collection of experimentally verified mammalian protein complexes to reveal the corresponding cliques complexes from SDMN. The interaction networks were generated by Cytoscape [47]. The pathway enrichment analysis was performed by Integrated Pathway Resources, Analysis and Visualization System (iPAVS) [48]. It is an integrated biological pathway database designed to support pathway discovery in systems biology research of schizophrenia. The analytic flowchart of this study is shown as Fig. 1.

\section{Results}

Schizophrenic genes with differential methylation and expression

There are 1689 SDMGs with $p$-value $<0.05$ identified as listed in Additional file 1. The SDMGs and SCZCGs 


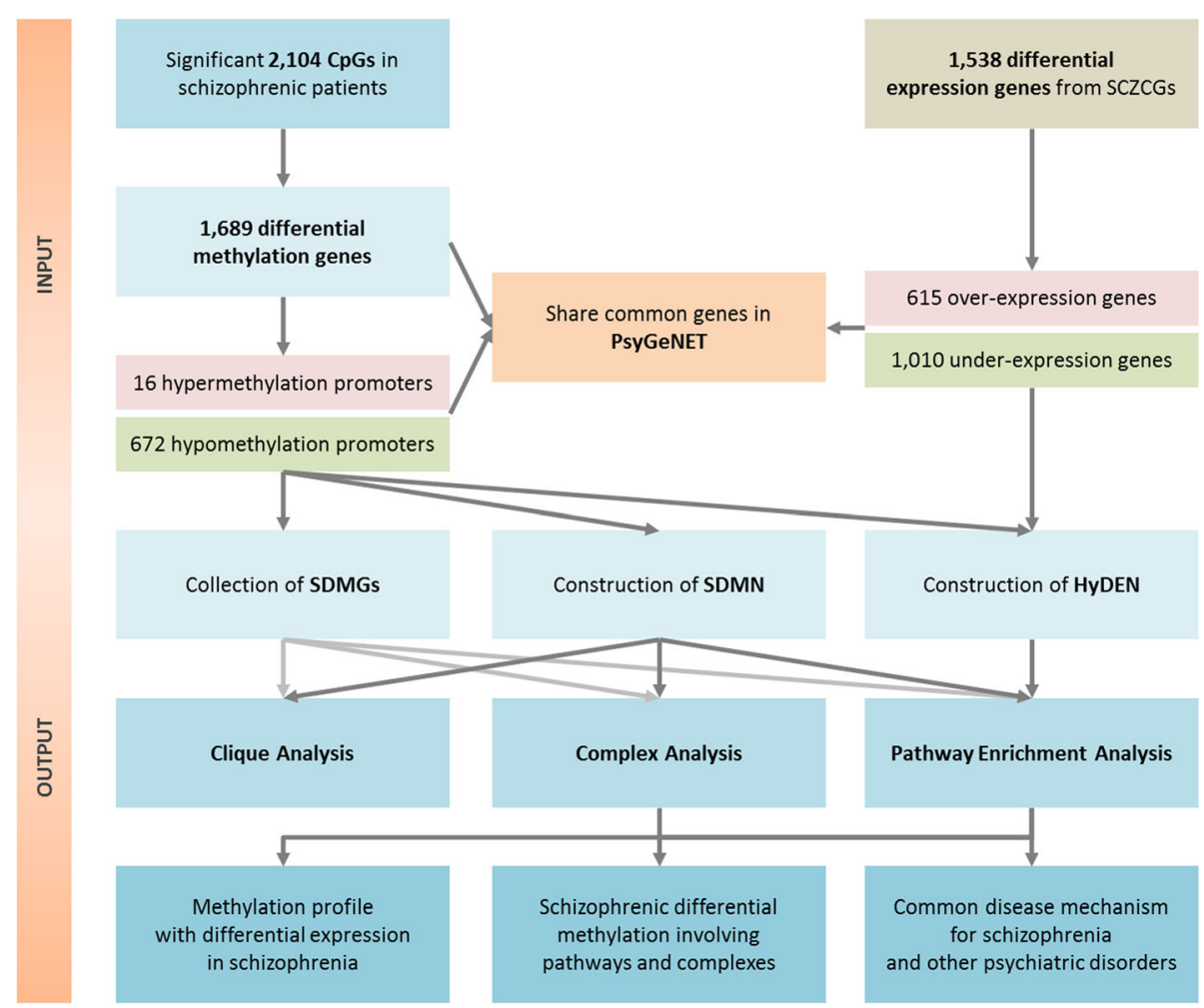

Fig. 1 Flowchart for the analysis and exploration of disease methylation profiles and related pathways. The differentially methylated genes and differentially expressed genes were selected to construct the SDMN and HyDEN. The PsyGeNET also shares disease marker genes with the list of SDMGs and SCZCGs. By the analysis of cliques, functional complexes and enriched pathways, we (a) studied the methylation profiles with differential gene expression in schizophrenia, (b) discovered related pathways from enrichment analysis of schizophrenic differential methylation, and (c) identified shared disease mechanisms for schizophrenia and other major psychiatric disorders

have 123 overlapped genes. The result illustrates the differential methylation and expression gene profile for schizophrenia (Additional file 1).

There are $688(39.6 \%)$ genes (16 hypermethylation/ 672 hypomethylation, ratio $2.38 \%$ ) differentially methylated in promotor regions from 1869 schizophrenic differentially methylated genes. 639 (36.9\%) genes (24 hypermethylation/615 hypomethylation, ratio 3.90\%) are differentially methylated in introns. 481 (27.7\%) genes (23 hypermethylation/458 hypomethylation, ratio $5.02 \%$ ) are differentially methylated in exons. The Venn diagram revealed that the most differential methylation genes are in promotor regions (39.6\%) and least differentially methylated in exons $(27.7 \%)$ of the schizophrenic methylation profile on specific gene location. The very different methylation profile in promotor regions may an etiology of schizophrenia. Previous studies have shown evidences of DNA methylation profile of several common genetic loci in schizophrenia. In this study, the hypomethylation of promotors was involved in schizophrenia. There is one gene (DAAM1) which was hypermethylated throughout promotors, introns and exons. The Venn diagram is shown as Fig. 2.

\section{Topological analysis of SDMN}

The schizophrenic differential methylation network (SDMN) was generated by QQPPI and genetic interactions in Pathway Commons Database of SDMGs. Among SDMGs, there were 10 hypermethylated genes in SDMN including GNA13, CAPNS1, GABPB2, GIT2, LEFTY1, NDUFA10, MIOS, MPHOSPH6, PRDM14 and RFWD2. The hypermethylated genes are distributed throughout the SDMN and might be important in the regulatory inhibition of gene expression in schizophrenia. The inferring interaction network is shown in Fig. 3.

The 10 schizophrenic hypermethylated genes discovered by SDMN are associated with biological functions such as cell structure, energy metabolism, mitochondrial function, GABA metabolism, signaling transduction and zinc fingers. These schizophrenic hypermethylated genes may have vital roles in the etiology of schizophrenia. For example, GNA13, a candidate disease marker of schizophrenia according to GWAS studies [49], effects brain microstructure and maintenance of the white matter. It plays a crucial role for G-protein signaling and neurodevelopment [50]. Expression of CAPNS1 gene with signal transductions of calcium-dependent activity changes in 


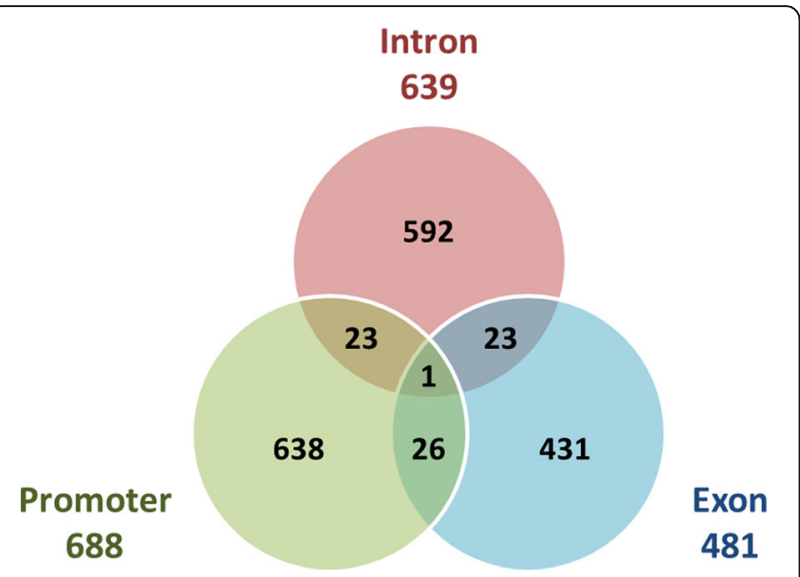

Lists contain 1734 unique elements

Fig. 2 The Venn diagram for the distribution of methylation sites in promoters, introns or exons. This Venn diagram compared the numbers of methylation sites in promoters, introns and exons. There are 639 genes that are differentially methylated in intron, 688 genes in promoters and 481 genes in exons. Genes which have been more than one regions are 23 (intron and promoter), 23 (intron and exon) and 26 (promoter and exon), respectively. DAAM1 is differentially methylated is in promoter, intron and exon regions

schizophrenia [51, 52]. It also significantly contributes to platelet activity and thrombosis [53], which may contribute to the increased risk of thrombogenesis in schizophrenic patients [2]. From the interaction aspect of neurotransmission, calpain-mediated break down of fGAD65 results in decreased level of the GABA synthesis which leads to reduced GABA neurotransmission [54]. The mTOR has a role mediated by Wnt signaling pathway in the neuropathology of schizophrenia [55]. LEFTY1 gene encodes a member of the TGF- $\beta$ family of proteins. Genetic variants in TGFB1 gene affect susceptibility to schizophrenia. TGF- $\beta$ signaling might be a valid link contributing to schizophrenia patients $[56,57]$. NDUFA10 has been contributed to the abnormalities of mitochondrial function in schizophrenia [58]. Also, the change of mitochondrial gene in respiratory electron transport chain responses to the exposure of antipsychotics [59].

Previous studies have validated the relationships between the hypermethylated genes and schizophrenia, yet, little is known about how methylation profile modulate the disease phenotype. With the analysis of SDMN, we could investigate the relationship between the hypermethylated genes and epigenetic mechanism in which the future experimental validation were needed. It may be one of the major disease mechanisms of schizophrenia.

The following hypermethylated genes are associated to schizophrenia susceptibility for the first time in this study, and have not been experimentally validated.
PRDM14, a member of PRDM family and zinc finger proteins involving transcriptional regulators decrease [60]. Another gene, RFWD2 (COP1) is a cancer suppressor and effects human $\beta$-Cell Insulin Secretion [61]. Loss of COP1 expression may contribute to tumorigenesis and regulate the expression of tumor suppressor TP53 $[18,62,63]$. RFWD2 may indirectly influence the etiology for schizophrenia. MPHOSPH6 is related to the pathways in Sertoli-Sertoli Cell Junction Dynamics and Deadenylation-dependent mRNA decay; it is in a RNA exosome complex and is genomewide associated with SNP clusters in schizophrenia [64]. MPHOSPH6 also interacts with TP53, which has imporatnt biological functions in schizophrenia pathology [65]. GABPB2 has not yet established relationships with schizophrenia. However, GABPB2 may be involved in the nuclear control of mitochondrial function which is important to the pathogenesis for schizophrenia [2]. The above genes not only influence the genetic methylation levels, but also the gene expression.

\section{Hypermethylated genes interact with differential expression network (HyDEN)}

The DNA hypermethylation could disable the gene function and decrease the gene expression level in many disease mechanisms, hence the relatioships between hypermethylated genes and differential expression was studied. All hypermethylated genes (or promoters) were collected and constructed into the HyDEN with the Level 1 PPIs of the encoded proteins.

The interactions in HyDEN may implicate important regulation mechanisms in schizophrenia. In Fig. 4, the schizophrenic hypermethylated genes (yellow colored) linked to differential expression genes (red colored for over-expressed and green colored for under-expressed). The figure indicates the majority of hypermethylated genes are linked to under-expression of schizophrenic candidate genes. Only MPHOSPH6 interacts with overexpressed RPS14. The HyDEN is composed of the hypermethylated genes in promotor regions and the interacting SCZCGs. Hypermethylated genes including RAMP1, CDK13 and PHACTR3, suppressed the expression of ATF3. Hypermethylated gene GABPB2 suppressed the expression of SEZ6L2, GLA, PRKACA and MAGED2. Hypermethylated gene CAPNS1 suppressed APEX1. From the perspective of genetic interactions of DNA methylation and gene expression, HyDEN may have demonstrated the core disease mechanism of schizophrenia.

\section{Biological pathways with differentially methylated schizophrenic genes}

Based on the topological analysis, the pathway enrichment analysis and underlying biological functions from 


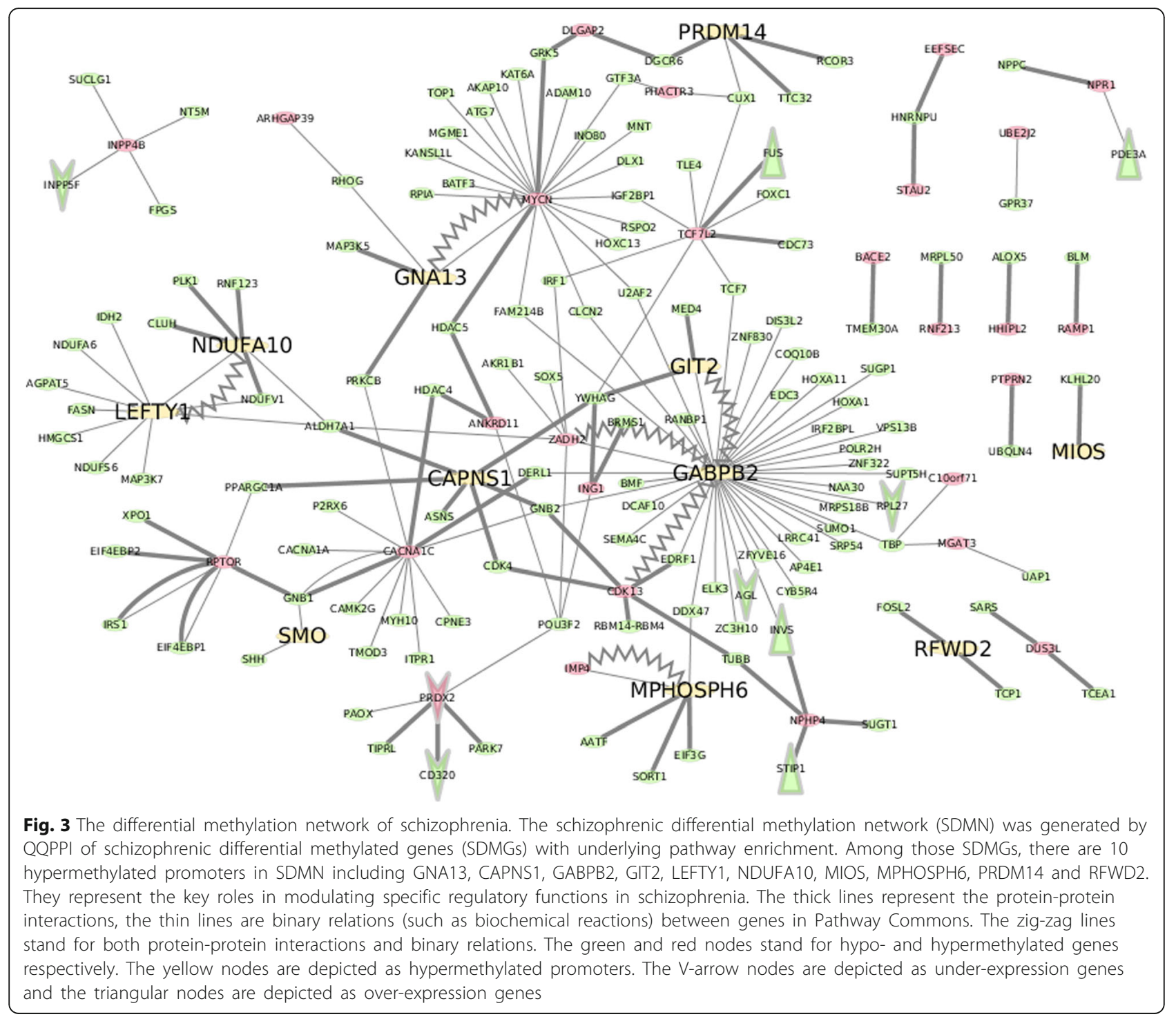

differential methylation profile are proposed. There are 8510 pathways (out of the 34,661 pathways listed in Pathway Commons Database) which contain SDMGs.

There are 29 corresponding pathways with FDR-adjust $p$-value $<0.05$ found in enrichment analysis from SDMGs which may implicate the underlying disease mechanisms and characteristics for schizophrenia under the regulatory role of SDMGs. Top ranked pathways with FDR $p$-value $<0.05$ are TGF_beta_receptor, Pyrimidine metabolism, metabolic pathways, WNT pathway, folate biosynthesis, nicotinate and nicotinamide metabolism and purine metabolism. The pathway enrichment analysis may indicate the biological functions influenced by SDMGs. It could reveal the potential disease mechanism and novel therapeutic strategy for schizophrenia. These pathways are listed in Additional file 2_A. In order to explore the mechanism influenced by methylated promotors, the enriched pathways are listed in
Additional file 2_B. For the investigation of how hypermethylating SDMGs regulate the corresponding pathways, the enriched pathways influenced by the hypermethylating SDMGs with Level 1 PPIs are listed in Additional file 2_C.

The top ranked pathways in Additional file 2_C, such as TNF alpha, PDGFR-beta signaling, TGF beta Receptor, VEGFR1 and VEGFR2 signaling, regulation of telomerase, hepatocyte growth factor receptor signaling, ErbB1 downstream signaling and mTOR signaling pathways, may be the key players in the symptoms of schizophrenia. Among these pathways, tumor necrosis factor alpha (TNF- $\alpha$ ) is a cytokine product. Its primary role is the regulation of immune cells with biological functions of apoptotic cell death, and inhibition of tumorigenesis and viral replication. Dysregulation of TNF- $\alpha$ production may causes negative symptoms of psychosis and schizophrenia 


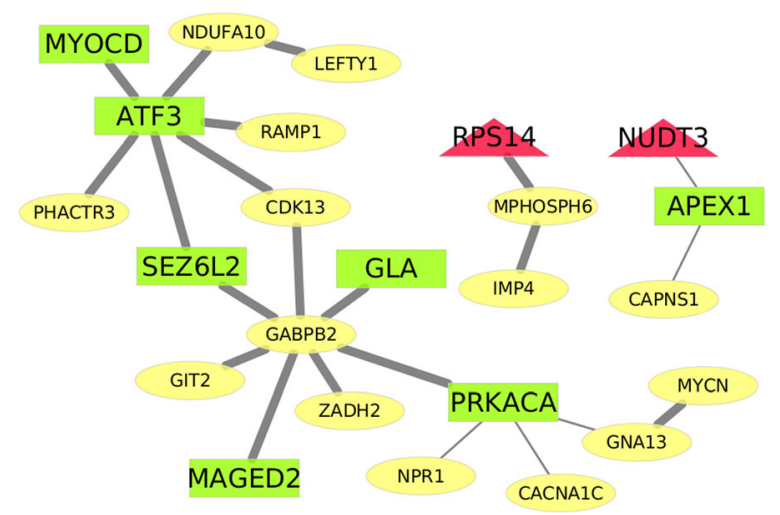

Fig. 4 Hypermethylation to differential expression network. The hypermethylation to differential expression network (HyDEN) was constructed by hypermethylated promoters, SCZCGs and their Level one PPIs. The schizophrenic hypermethylated promoters, which interacting with differential expression genes, indicate the majority of hypermethylated genes suppressed the expression SCZCGs. The red triangular nodes are depicted as over-expressed genes and the green rectangle nodes are depicted as under-expressed genes, the yellow eclipse nodes are hypermethylated genes. The thick lines are PPIs. The thin lines are binary relations (e.g. biochemical reactions) between genes listed in Pathway Commons Databases

$[66,67]$. Platelet-derived growth factor receptors (PDGF-R) are cell surface tyrosine kinase receptors. Its subunits $-\mathrm{A}$ and $-\mathrm{B}$ are important factors which regulate cell proliferation, cellular differentiation, cell growth and neuronal development. The genes for platelet-derived growth factor beta (PDGFB) and PDGFB receptor (PDGFBR) may be important in the pathology of schizophrenia through interacting with the DRD2/DRD4 and NMDA receptors [68]. It should be noticed that PDGFRB mRNA transcripts are significantly increased in postmortem brains of schizophrenic patients [69].

The Wnt pathway and the canonical pathway are targets of antipsychotic treatments. The most consistent abnormalities associated with antipsychotic response are Akt1, glycogen synthase kinase3beta and beta-catenin pathways [70]. VEGF is associated with inflammation reaction. Its serum levels are related to prefrontal cortex abnormalities in schizophrenia [71]. Shortened telomere length is found in unremitted schizophrenic patients [72]. A significant decrease is noted in telomerase activity among individuals with schizophrenia [73]. Hyperactivity of the epidermal growth factor receptor family (ErbB) is implicated in the pathophysiology of schizophrenia. ErbB receptor tyrosine kinases may be novel therapeutic targets for schizophrenia. Deficits in ErbB signaling pathway might contribute to the neurological development of psychiatric diseases [74, 75]. ErbB inhibitors appear to have anti-dopaminergic actions to alleviate behavioral symptoms in animal models for schizophrenia [76]. The mTOR signaling cascade involves in the regulation of neuronal morphology and synaptic plasticity. Disrupted mTOR signaling cause impaired function of protein synthesis in schizophrenia [55]. Those involved pathways are significantly found by enrichment analysis of differential methylated genes with hypermethylated genes and promotor regions. They could reveal the pathology and disease mechanism for schizophrenia.

\section{Shared disease mechanisms of schizophrenia and other psychiatric disorders}

In order to compare the common disease mechanism between schizophrenia and the different psychiatric disorder, a knowledge platform for the exploratory analysis of psychiatric diseases and their associated genes known as PsyGeNET [47] was analyzed to search for differential methylated genes of schizophrenia. The platform focuses mainly on major depression, and alcohol and cocaine additions.

There are 308 overlapped genes in PsyGeNET from 1538 SCZCGs, and 64 overlapped genes from 688 SDMGs in promotors and PsyGeNET. The selected genes are listed in Additional file 3. Of the 308 overlapped genes from PsyGeNET and SCZCGs, the most frequently appeared ( $>=7$ psychiatric disorders) genes are DRD2, TPH2, S100B, GAD1, DTNBP1, GFAP and CARTPT among different psychiatric disorders such depressive disorder, bipolar disorder, alcoholism, cocaine-related disorders and suicide. It reveals that those vital mental illnesses in PsyGeNET may share common marker genes with the SCZCGs. The molecular mechanisms for those genes involves Gprotein receptor activity, tryptophan metabolism, cell cycle progression and differentiation, GPCR signaling, glutamate metabolism, neurotransmitter release, actin cytoskeleton reorganization, structural molecule activity and energy balance. They are also pivotal genes in the development of schizophrenia, bipolar disorder, major depressive disorder, cocaine dependence and Alzheimer's disease [77-84].

Of all 64 overlapped genes from the SDMGs and PsyGeNET, the most frequent appeared genes are NR3C2, HDAC5, FTO, XBP1, RNF41 and NDUFV1. Those are pivotal genes for alcoholism, schizophrenia, major depression, bipolar disorder [85-90]. The involved biological functions of transcription factor activity, steroid hormone receptor activity, transcriptional regulation, cell cycle progression and developmental events, oxidative RNA demethylase activity and NADH dehydrogenase (ubiquinone) activity. The shared pathways are signaling by GPCR, immune system and metabolism. 
Protein complexes and genetic interactions in SDMGs and SCZCGs

In order to understand the involved protein complexes in schizophrenia of how SDMGs interact with the expression level of SCZCGs, we searched CORUM for the potential protein complexes responsible for the regulation and epigenetic mechanism in schizophrenia. CORUM is a database that provides information of experimentally characterized protein complexes from human resource to predict the occurrence of protein complexes in different polygenetic groups [91]. We analyzed the gene groups from SDMGs and SCZCGs, and searched against CORUM to find the potential protein complexes related to the etiology of schizophrenia from alteration of DNA methylation. The protein complexes involved in SDMGs and SCZCGs are listed in Additional file 4 .

The most important protein complexes involved in SDMGs and SCZCGs may include Nop56p-associated pre-rRNA complex, ribosome related subunit, mitochondrial respiratory chain complex I, TFTC complex and PCAF complex. The biological functions of those complexes are associated with ribosome biosynthesis, mitochondrial dysfunction and pre-rRNA processing. However, the top ranked complexes represented in SDMGs including SMCC complex, Mediator complex, Nop56p-associated pre-rRNA complex, CDC5L complex, CF IIAm complex and $55 \mathrm{~S}$ mitochondrial ribosome complex. These complexes are translated by aberrant SDMGs to perform specific protein functions, which might be the potential molecular mechanism in epigenetic regulation for schizophrenia. The inheritable alterations of these complexes might explain the roles of hereditary factors in the etiology of schizophrenia with DNA methylation [92].

\section{Discussion}

\section{The roles of DNA differential methylation in} schizophrenia

External factors such as environmental stress are known to cause the onset of schizophrenia. Exposure to stress induces stable changes by transcriptional dysfunction, resulting in aberrant changes of genetic expression, neural circuit functions and ultimately, behavior changes and disease symptoms [93]. Epigenetic factors such as aberrant DNA methylation have important roles in regulating gene expression [94]. Epigenetic changes may be one of the pivotal features of many human mental disorders. In cancer etiology, the promoter hypermethylation also plays a major role by aberrant transcription of critical regulator genes such as tumor suppressor genes with the implications for the hypomethylation factors in the novel treatment strategy of cancer [95].
Epigenetic mechanism produces DNA methylation which alter gene expression without altering underlying DNA sequence. Epigenetic changes may be passed on for multiple generations by cell division [96]. Evidences of linkage analysis in schizophrenic family suggest a hereditary susceptibility [97]. The methylation of DNA confers long-term epigenetic silencing which could be reprogrammed by demethylation of DNA repair [98]. It is implicated that the epigenetic change, especially from the differentially expression genes, regulate the methylation of SDMGs and the production of corresponding protein complexes.

Methylation in the transcribed region is often correlated with expression, and high levels of gene expression is often associated with low promoter methylation [99-101]. The hypermethylated genes are usually under-expressed. The relationships between the hypermethylated genes and the differential expression genes in schizophrenia (HyDEN) reveals that the hypermethylated promotors are not necessarily associated with their differential expression level, instead, the interacting genes with hypermethylated SDMGs are mostly under-expressed SCZCGs. These gene interactions may be important in the regulation of corresponding pathways and biological functions for schizophrenia.

Our results suggest that the hypomethylated genes are predominant in schizophrenia. Reducing hypomethylation of SDMGs or SCZCGs could be a novel therapeutic treatment method for schizophrenia. There might be the protective factors as per the etiology of cancer [18], in which most promotors are hypermethylated. Some hypermethylating agent, such as vitamin B1, could induce up-regulation of methyltransferase and reversion of hypomethylation as an adjuvant treatment in schizophrenia [102]. It has postulated that deficiency of vitamin B1 may result in genetic methylation and biochemical lesion relating to neurotransmitter metabolism in brain, leading to psychotic manifestations [103].

DNA methylation and histone modifications can alter genome functions under exogenous influences. These heritable changes in gene expression may be more than just changes of DNA sequence. The ribosome-associated PPI network was constructed with SDMGs combined with SCZCGs (Fig. 5), and the topological analysis revealed that groups of hypomethylated genes XPO1, HNRNPU, IGSF8, SND1 and FUS which ingeniously interact with over-expressed genes NCL, FAU and HNRPNPM, as well as with under-expressed genes: EED, TP53, RNF2, HUWE1, SLC25A5 and FN1. It reveals the regulatory role and epigenetic mechanism in schizophrenia with novel targets of therapeutic agents. In the analysis of 


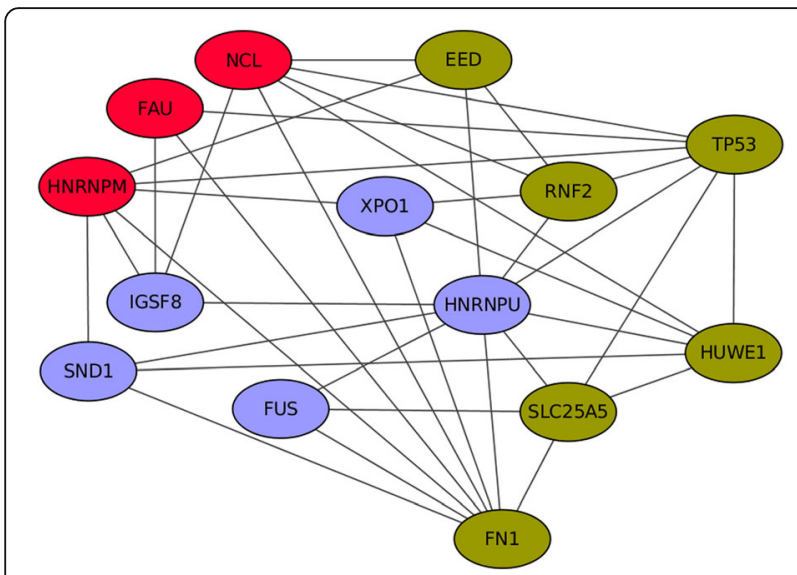

Fig. 5 The ribosome-associated PPI network. The ribosomeassociated PPI network constructed with SDMGs and SCZCGs revealed the regulatory role of epigenetic mechanism in schizophrenia. The red and green nodes are depicted as over- and under-expressed SCZCGs, respectively. The purple nodes are hypomethylated promoters. The ribosome biosynthesis is likely to be regulated by hypomethylated SDMGs which interacts with differentially expressed SCZCGs. The regulation of ribosome expression by the hypomethylated promoters may be crucial in the pathogenesis for schizophrenia

HyDEN and the regulatory hypomethylated genes in ribosome, there appears to have interactions between differential DNA methylation and differential expression levels. There seems to be interactions among the regulatory factors at different stages of gene expression and genetic inheritance.

In published works, the hypermethylated genes in HIPPO signaling and MAPK signaling pathways have been observed in schizophrenic patients. Hypomethylated promoters in CREB signaling, dopamineDARPP32 feedback in cAMP signaling and Ephrin receptors have also been noted. CREB3L2 in differential expression genes was under-expressed; CREB3 and PPP1R1B (DARPP32) were hypomethylated in SDMGs. Another example of hypomethylation in FAM63B was found in bipolar disorder. FAM63B was an epigenetic risk gene for schizophrenia and bipolar disorder [104]. However, FAM65B, FAM64A and FAM69A have been reported as hypomethylated in SDMGs. LRRTM1 hypomethylation in the promoter was reported as a risk factor for the development of schizophrenia [105]. LRRTM2 was over-expressed in $\mathrm{Wu}$ et al. [20]; LRRTM4 was under-expressed in Huang, et al. [2]. Antipsychotic such as quetiapine decreased the DNA methylation level of the promoter region of SLC6A4. Mood stabilizers could also reverse the hypermethylation process of CpG sites of SLC6A4 to be hypomethylated in bipolar disorder [106, 107]. However, SLC6A9 was under-expressed gene in Huang et al. [2].

\section{Epigenetics in protein complexes for schizophrenia}

For the understanding of epigenetic mechanism for schizophrenia, the potential protein complex interactions reveal the relationships between the genomic and the environmental factors during disease development. Among the top ranked complexes, there are reported complexes by literature associated with epigenetic mechanism for schizophrenia. The mediator complex is a key role in regulation of transcription of RNA polymerase II. It was characterized as TRAP complex that facilitates transcriptional activation by thyroid hormone receptor [108, 109]. The mutations in TRAP230 could result in the attenuated functions by p53 activation to target RNA polymerase II [110] and transcriptional activators by TRAP/Mediator complex correlate with the development of schizophrenia [111, 112]. CDC5L complex is essential for the catalytic step of pre-mRNA splicing [113]. Epigenetic factors such as stress induced prefrontal cognitive dysfunction, schizophrenic patients with DISC1 mutations are vulnerable to the effects of stress by increasing cAMP levels [114]. DISC1 network of PPIs involved CDC5L indicates the protein or complexes that have been linked to schizophrenia [115]. It implicates that the DISC-1 associated CDC5L complex may explain the epigenetic mechanism in stressinduced prefrontal dysfunction in schizophrenia.

The alteration of mitochondria and dysregulation of energy metabolism in postmortem brain samples may contribute to implication of schizophrenia [116, 117]. The genetic interactions among mitochondrial genes and many under-expressed SCZCGs indicate the genetic predisposition of mitochondria dysfunction in schizophrenia. The genetic interactions between mitochondria and schizophrenia may be revealed by the DRD2NDUFS7 and the FLNA-ARRB2 interactions [2]. In this study, NDUFA10 in HyDEN has found to be associated with the abnormalities of mitochondrial function in schizophrenia [58]. It plays a key role in respiratory electron transport chain responded to the exposure of antipsychotics [59]. NDUFA10 mutation causes mitochondrial complex I deficiency. It is associated with the progressive neurodegenerative disease such as Leigh syndrome [118], which possibly shares the same etiopathogeny with schizophrenia [119]. The mechanism involving NDUFA10 could be novel targets for schizophrenic therapeutic treatments.

\section{Conclusions}

In order to understand the regulatory role of SDMGs, the epigenetic profiles of DNA differential methylation in schizophrenic postmortem brain samples was analyzed in this study. Aberrant DNA methylation have been associated with various neurodevelopmental and 
neuropsychiatric disorders. The interactions between SDMGs and SCZCGs may be part of the underlying disease mechanism of schizophrenia, with the DNA differential methylation influencing the levels of gene expression. It is implicated that corresponding complexes and pathways are pivotal and for the disease mechanism for schizophrenia.

The SDMN was extended with the Level 1 PPIs of SDMGs to explore the potential protein complexes and biochemical pathways of the disease mechanism and therapeutic options for schizophrenia. The SDMGs interacted with SCZCGs in a coordinated fashion in schizophrenia. The majority of hypermethylated SDMGs were associated with the under-expression of SCZCGs, indicating the regulatory role of hypermethylated promotors to suppress SCZCGs and the consequent suppressiong of coppresponding pathways such as TNF alpha, PDGFR-beta signaling, TGF beta Receptor, VEGFR1 and VEGFR2 signaling, regulation of telomerase, hepatocyte growth factor receptor signaling, ErbB1 downstream signaling and mTOR signaling. The dysfunction of these pathways may be the causes of schizophrenia. Altered DNA methylation, including both hyper and hypo, appears to play a complementary role in schizophrenia development. The predominant hypomethylation of SDMG promoters is distinct from the characteristic predominantly hypermethylation of promotors in cancers. Increasing methylation of these abnormally hypomethylated promotors could be a potential therapeutic option for schizophrenia.

\section{Additional files}

Additional file 1: Differentially methylated genes with differential expression databases. (XLSX $170 \mathrm{~kb}$ )

Additional file 2: Pathway enrichment analysis of SDMGs, hypermethylating SDMGs and SDMGs with level 1 PPI. (XLSX 576 kb)

Additional file 3: The shared common genes of 688 SDMG promoters and PsyGeNET. (XLSX 20 kb)

Additional file 4: The protein complexes involved in SDMGs and SCZCGs. (XLSX 27 kb)

\section{Acknowledgements}

We would like to give special thanks to Beitou Branch, Tri-Service General Hospital, National Defense Medical Center for providing space and facilities for necessary computing machine and discussion. We are grateful that Information Technology Center, Kainan University provided highperformance computing resources. We also thank T. T. H. Tsao for polishing the English of this paper. This research project was funded by MOST grant (Project ID: MOST-105-2221-E-424-004-).

\section{Declaration}

This article has been published as part of BMC Medical Genomics Volume 9 Supplement 3, 2016. 15th International Conference on Bioinformatics (INCOB 2016): medical genomics. The full contents of the supplement are available online https://bmcmedgenomics.biomedcentral.com/articles/supplements/ volume-9-supplement-3

\section{Funding}

Publication charges for this article were funded by the first author's institution.

\section{Availability of data and materials}

All data generated or analyzed during this study are included in this published article.

\section{Authors' contributions}

$\mathrm{KCH}$ interpreted the results, drafted the manuscript and contributed to the design of the bioinformatics analysis tools. SAL programmed the bioinformatics analysis tools and carried out data analysis. SAL conceived the study and participated in coordination and management of the research project. SAL helped to generate figures, and $\mathrm{KCH}$ managed to process the additional files. Both authors read and approved the final manuscript.

\section{Competing interests}

The authors declare that they have no competing interests.

\section{Consent for publication}

Not applicable.

Ethics approval and consent to participate

Not applicable.

\section{Author details}

${ }^{1}$ Department of Information Management, Kainan University, Taoyuan, Taiwan. 'Department of Psychiatry, Beitou Branch, Tri-Service General Hospital, National Defense Medical Center, Taipei, Taiwan. ${ }^{3}$ Department of Nursing, Ching Kuo Institute of Management and Health, Keelung, Taiwan.

Published: 5 December 2016

\section{References}

1. Allen NC, et al. Systematic meta-analyses and field synopsis of genetic association studies in schizophrenia: the SzGene database. Nat Genet. 2008; 40(7):827-34.

2. Huang KC, et al. Transcriptome alterations of mitochondrial and coagulation function in schizophrenia by cortical sequencing analysis. BMC Genomics. 2014;15 Suppl 9:S6.

3. Akbarian S. Epigenetic mechanisms in schizophrenia. Dialogues Clin Neurosci. 2014;16(3):405-17.

4. Li Y, et al. Genome-wide methylome analyses reveal novel epigenetic regulation patterns in schizophrenia and bipolar disorder. Biomed Res Int. 2015;2015:201587.

5. Montano C, et al. Association of DNA Methylation Differences With Schizophrenia in an Epigenome-Wide Association Study. JAMA Psychiat. 2016:73(5):506-14

6. Nishioka M, et al. DNA methylation in schizophrenia: progress and challenges of epigenetic studies. Genome Med. 2012;4(12):96.

7. Rivollier F, et al. Epigenetics of schizophrenia: a review. Encéphale. 2014; 40(5):380-6.

8. Teroganova N, et al. DNA methylation in peripheral tissue of schizophrenia and bipolar disorder: a systematic review. BMC Genet. 2016;17:27.

9. Shimabukuro $M$, et al. Global hypomethylation of peripheral leukocyte DNA in male patients with schizophrenia: a potential link between epigenetics and schizophrenia. J Psychiatr Res. 2007;41(12):1042-6.

10. Misiak $B$, et al. Lower LINE-1 methylation in first-episode schizophrenia patients with the history of childhood trauma. Epigenomics. 2015;7(8): 1275-85.

11. Kinoshita $\mathrm{M}$, et al. DNA methylation signatures of peripheral leukocytes in schizophrenia. Neuromolecular Med. 2013;15(1):95-101.

12. Grayson DR, Guidotti A. The dynamics of DNA methylation in schizophrenia and related psychiatric disorders. Neuropsychopharmacology. 2013;38(1): 138-66.

13. Schilling E, El Chartouni C, Rehli M. Allele-specific DNA methylation in mouse strains is mainly determined by cis-acting sequences. Genome Res. 2009;19(11):2028-35.

14. Dong $\mathrm{E}_{\text {, et }}$ al. Behavioral and molecular neuroepigenetic alterations in prenatally stressed mice: relevance for the study of chromatin remodeling properties of antipsychotic drugs. Transl Psychiatry. 2016;6:e711. 
15. O'Shea KS, McInnis MG. Neurodevelopmental origins of bipolar disorder: iPSC models. Mol Cell Neurosci. 2016;73:63-83.

16. Jaffe $A E$, et al. Mapping DNA methylation across development, genotype and schizophrenia in the human frontal cortex. Nat Neurosci. 2016;19(1):40-7.

17. Hannon E, et al. Methylation QTLs in the developing brain and their enrichment in schizophrenia risk loci. Nat Neurosci. 2016;19(1):48-54

18. Huang KC, et al. Analysis of schizophrenia and hepatocellular carcinoma genetic network with corresponding modularity and pathways: novel insights to the immune system. BMC Genomics. 2013;14 Suppl 5:S10.

19. Lee $S A$, et al. Construction and analysis of the protein-protein interaction networks for schizophrenia, bipolar disorder, and major depression. BMC Bioinformatics. 2011;Suppl 13:S20.

20. Wu JQ, et al. Transcriptome sequencing revealed significant alteration of cortical promoter usage and splicing in schizophrenia. PLoS One. 2012;7(4): e36351.

21. Sun J, et al. Schizophrenia gene networks and pathways and their applications for novel candidate gene selection. PLoS One. 2010;5(6): e11351.

22. Beveridge NJ, et al. Schizophrenia is associated with an increase in cortical microRNA biogenesis. Mol Psychiatry. 2010;15(12):1176-89.

23. Strimmer K. fdrtool: a versatile R package for estimating local and tail areabased false discovery rates. Bioinformatics. 2008;24(12):1461-2.

24. Lee SA, et al. POINeT: protein interactome with sub-network analysis and hub prioritization. BMC Bioinformatics. 2009;10:114.

25. Cerami EG, et al. Pathway Commons, a web resource for biological pathway data. Nucleic Acids Res. 2011;39(Database issue):D685-90.

26. Bader GD, et al. BIND-The Biomolecular Interaction Network Database. Nucleic Acids Res. 2001;29(1):242-5.

27. Stark C, et al. BioGRID: a general repository for interaction datasets. Nucleic Acids Res. 2006;34(Database issue):D535-9.

28. Mattingly CJ, et al. The Comparative Toxicogenomics Database (CTD): a resource for comparative toxicological studies. J Exp Zool A Comp Exp Biol. 2006;305(9):689-92.

29. Orchard $\mathrm{S}$, et al. Protein interaction data curation: the International Molecular Exchange (IMEx) consortium. Nat Methods. 2012;9(4):345-50.

30. Mathivanan S, et al. An evaluation of human protein-protein interaction data in the public domain. BMC Bioinformatics. 2006;7 Suppl 5:S19.

31. Caspi R, et al. The MetaCyc database of metabolic pathways and enzymes and the BioCyc collection of pathway/genome databases. Nucleic Acids Res. 2012;40(Database issue):D742-53.

32. Orchard S, et al. The MIntAct project-IntAct as a common curation platform for 11 molecular interaction databases. Nucleic Acids Res. 2014;42(Database issue):D358-63.

33. Kanehisa $\mathrm{M}$, et al. Data, information, knowledge and principle: back to metabolism in KEGG. Nucleic Acids Res. 2014;42(Database issue):D199-205.

34. Kandasamy K, et al. NetPath: a public resource of curated signal transduction pathways. Genome Biol. 2010;11(1):R3.

35. Thomas PD, et al. PANTHER: a browsable database of gene products organized by biological function, using curated protein family and subfamily classification. Nucleic Acids Res. 2003:31(1):334-41.

36. Hornbeck PV, et al. PhosphoSitePlus, 2014: mutations, PTMs and recalibrations. Nucleic Acids Res. 2015:43(Database issue):D512-20.

37. Schaefer CF, et al. PID: the Pathway Interaction Database. Nucleic Acids Res. 2009:37(Database issue):D674-9.

38. Croft D, et al. Reactome: a database of reactions, pathways and biological processes. Nucleic Acids Res. 2011;39(Database issue):D691-7.

39. Jewison T, et al. SMPDB 2.0: big improvements to the Small Molecule Pathway Database. Nucleic Acids Res. 2014:42(Database issue):D478-84.

40. Wingender $\mathrm{E}$. The TRANSFAC project as an example of framework technology that supports the analysis of genomic regulation. Brief Bioinform. 2008;9(4):326-32.

41. Hsu SD, et al. miRTarBase: a database curates experimentally validated microRNA-target interactions. Nucleic Acids Res. 2011;39(Database issue): D163-9.

42. Law V, et al. DrugBank 4.0: shedding new light on drug metabolism. Nucleic Acids Res. 2014;42(Database issue):D1091-7.

43. Thiele I, et al. A community-driven global reconstruction of human metabolism. Nat Biotechnol. 2013;31(5):419-25.

44. Kutmon M, et al. WikiPathways: capturing the full diversity of pathway knowledge. Nucleic Acids Res. 2016;44(D1):D488-94.
45. Gutierrez-Sacristan A, et al. PsyGeNET: a knowledge platform on psychiatric disorders and their genes. Bioinformatics. 2015;31(18):3075-7.

46. Ruepp A, et al. CORUM: the comprehensive resource of mammalian protein complexes. Nucleic Acids Res. 2008:36(Database issue):D646-50.

47. Shannon P, et al. Cytoscape: a software environment for integrated models of biomolecular interaction networks. Genome Res. 2003;13(11):2498-504.

48. Sreenivasaiah PK, et al. IPAVS: Integrated Pathway Resources, Analysis and Visualization System. Nucleic Acids Res. 2012;40(Database issue):D803-8.

49. Jia P, et al. Network-assisted investigation of combined causal signals from genome-wide association studies in schizophrenia. PLoS Comput Biol. 2012; 8(7):e1002587.

50. Sprooten $\mathrm{E}$, et al. Common genetic variants and gene expression associated with white matter microstructure in the human brain. Neuroimage. 2014;97: 252-61.

51. Maycox PR, et al. Analysis of gene expression in two large schizophrenia cohorts identifies multiple changes associated with nerve terminal function. Mol Psychiatry. 2009:14(12):1083-94.

52. Glatt SJ, et al. Comparative gene expression analysis of blood and brain provides concurrent validation of SELENBP1 up-regulation in schizophrenia. Proc Natl Acad Sci U S A. 2005;102(43):15533-8.

53. Tyagi T, et al. Altered expression of platelet proteins and calpain activity mediate hypoxia-induced prothrombotic phenotype. Blood. 2014;123(8): $1250-60$.

54. Buddhala C, et al. Calpain cleavage of brain glutamic acid decarboxylase 65 is pathological and impairs GABA neurotransmission. PLoS One. 2012;7(3): e33002.

55. Gururajan A, van den Buuse M. Is the mTOR-signalling cascade disrupted in Schizophrenia? J Neurochem. 2014;129(3):377-87.

56. Frydecka $D$, et al. Sex differences in TGFB-beta signaling with respect to age of onset and cognitive functioning in schizophrenia. Neuropsychiatr Dis Treat. 2015;11:575-84.

57. Frydecka $D$, et al. Genetic variants in transforming growth factor-beta gene (TGFB1) affect susceptibility to schizophrenia. Mol Biol Rep. 2013;40(10): 5607-14.

58. Park C, Park SK. Molecular links between mitochondrial dysfunctions and schizophrenia. Mol Cells. 2012;33(2):105-10.

59. Ji B, et al. A comparative proteomics analysis of rat mitochondria from the cerebral cortex and hippocampus in response to antipsychotic medications. J Proteome Res. 2009;8(7):3633-41.

60. Lin $M$, et al. RNA-Seq of human neurons derived from iPS cells reveals candidate long non-coding RNAs involved in neurogenesis and neuropsychiatric disorders. PLoS One. 2011;6(9):e23356.

61. Suriben R, et al. beta-Cell Insulin Secretion Requires the Ubiquitin Ligase COP1. Cell. 2015;163(6):1457-67.

62. Sawada G, et al. Loss of COP1 expression determines poor prognosisin patients with gastric cancer. Oncol Rep. 2013;30(4):1971-5.

63. Ni X, et al. Human p53 tumor suppressor gene (TP53) and schizophrenia: case-control and family studies. Neurosci Lett. 2005;388(3):173-8.

64. Sullivan PF, et al. Genomewide association for schizophrenia in the CATIE study: results of stage 1. Mol Psychiatry. 2008;13(6):570-84.

65. Stelzl U, et al. A human protein-protein interaction network: a resource for annotating the proteome. Cell. 2005;122(6):957-68.

66. Zeni-Graiff M. Peripheral immuno-inflammatory abnormalities in ultra-high risk of developing psychosis. Schizophr Res. 2016;176(2-3):191-5.

67. Turhan $\mathrm{L}$, et al. The role of tumour necrosis factor alpha and soluble tumour necrosis factor alpha receptors in the symptomatology of schizophrenia. Nord J Psychiatry. 2016;70(5):342-50.

68. Zai G, et al. Weak association of the platelet-derived growth factor beta (PDGFB) and PDGF receptor beta (PDGFRB) genes with schizophrenia and schizoaffective disorder. World J Biol Psychiatry. 2011;12(2):127-33.

69. Prabakaran S, et al. Mitochondrial dysfunction in schizophrenia: evidence for compromised brain metabolism and oxidative stress. Mol Psychiatry. 2004 9(7):684-97. 643.

70. Panaccione I, et al. Neurodevelopment in schizophrenia: the role of the wnt pathways. Curr Neuropharmacol. 2013;11(5):535-58.

71. Pillai $A$, et al. Association of serum VEGF levels with prefrontal cortex volume in schizophrenia. Mol Psychiatry. 2016;21(5):686-92.

72. Kota LN, et al. Shortened telomere in unremitted schizophrenia. Psychiatry Clin Neurosci. 2015;69(5):292-7.

73. Porton B, et al. Telomerase levels in schizophrenia: a preliminary study. Schizophr Res. 2008;106(2-3):242-7. 
74. Mizuno M, et al. Antipsychotic potential of quinazoline ErbB1 inhibitors in a schizophrenia model established with neonatal hippocampal lesioning. J Pharmacol Sci. 2010;114(3):320-31.

75. Iwakura $\mathrm{Y}$, Nawa $\mathrm{H}$. ErbB1-4-dependent EGF/neuregulin signals and their cross talk in the central nervous system: pathological implications in schizophrenia and Parkinson's disease. Front Cell Neurosci. 2013;7:4.

76. Mizuno M, et al. ErbB inhibitors ameliorate behavioral impairments of an animal model for schizophrenia: implication of their dopamine-modulatory actions. Transl Psychiatry. 2013;3:e252.

77. Edwards AC, et al. Evaluating the dopamine hypothesis of schizophrenia in a large-scale genome-wide association study. Schizophr Res. 2016;176(2-3): 136-40.

78. Gao J, et al. TPH2 gene polymorphisms and major depression-a metaanalysis. PLoS One. 2012;7(5):e36721.

79. Yelmo-Cruz S, Morera-Fumero AL, Abreu-Gonzalez P. S100B and schizophrenia. Psychiatry Clin Neurosci. 2013;67(2):67-75.

80. Hettema JM, et al. Association between glutamic acid decarboxylase gene and anxiety disorders, major depression, and neuroticism. Mol Psychiatry. 2006;11(8):752-62

81. Weickert CS, et al. Human dysbindin (DTNBP1) gene expression in normal brain and in schizophrenic prefrontal cortex and midbrain. Arch Gen Psychiatry. 2004;61(6):544-55

82. Chaves ML, et al. Serum levels of S100B and NSE proteins in Alzheimer's disease patients. J Neuroinflammation. 2010;7:6.

83. Webster MJ, et al. Glial fibrillary acidic protein mRNA levels in the cingulate cortex of individuals with depression, bipolar disorder and schizophrenia. Neuroscience. 2005;133(2):453-61.

84. Lohoff FW, et al. Genetic variants in the cocaine- and amphetamineregulated transcript gene (CARTPT) and cocaine dependence. Neurosci Lett. 2008;440(3):280-3

85. Tang L, et al. A NR3C2 haplotype increases the risk of alcoholism in schizophrenic patients in Han Chinese population. Psychiatry Res. 2015; 229(3):1057-8.

86. Iga J, et al. Altered HDAC5 and CREB mRNA expressions in the peripheral leukocytes of major depression. Prog Neuropsychopharmacol Biol Psychiatry. 2007:31(3):628-32.

87. Song $X$, et al. Fat-mass and obesity-associated gene polymorphisms and weight gain after risperidone treatment in first episode schizophrenia. Behav Brain Funct. 2014;10(1):35.

88. Jonsson $\mathrm{EG}$, et al. Association study of a functional promoter polymorphism in the XBP1 gene and schizophrenia. Am J Med Genet B Neuropsychiatr Genet. 2006;141B(1):71-5.

89. Kim S, et al. An E3 ubiquitin ligase, Really Interesting New Gene (RING) Finger 41, is a candidate gene for anxiety-like behavior and beta-carbolineinduced seizures. Biol Psychiatry. 2009;65(5):425-31.

90. Ben-Shachar D, Karry R. Sp1 expression is disrupted in schizophrenia; a possible mechanism for the abnormal expression of mitochondrial complex I genes, NDUFV1 and NDUFV2. PLoS One. 2007;2(9):e817.

91. Ruepp A, et al. CORUM: the comprehensive resource of mammalian protein complexes-2009. Nucleic Acids Res. 2010;38(Database issue):D497-501.

92. Roth $\mathrm{TL}$, et al. Epigenetic mechanisms in schizophrenia. Biochim Biophys Acta. 2009;1790(9):869-77.

93. Nestler EJ, et al. Epigenetic basis of mental illness. Neuroscientist. 2015 doi:10.1177/1073858415608147

94. Lim DHK, Maher ER. DNA methylation: a form of epigenetic control of gene expression. Obstet Gynecol. 2010;12(1):37-42.

95. Baylin SB. DNA methylation and gene silencing in cancer. Nat Clin Pract Oncol. 2005;2 Suppl 1:S4-S11

96. Bird A. Perceptions of epigenetics. Nature. 2007:447(7143):396-8.

97. Paunio $T$, et al. Linkage analysis of schizophrenia controlling for population substructure. Am J Med Genet B Neuropsychiatr Genet. 2009;150B(6):827-35

98. Reik W. Stability and flexibility of epigenetic gene regulation in mammalian development. Nature. 2007;447(7143):425-32.

99. Kass SU, Landsberger N, Wolffe AP. DNA methylation directs a timedependent repression of transcription initiation. Curr Biol. 1997;7(3):157-65

100. Jones PA. The DNA methylation paradox. Trends Genet. 1999;15(1):34-7.

101. Wagner JR, et al. The relationship between DNA methylation, genetic and expression inter-individual variation in untransformed human fibroblasts. Genome Biol. 2014;15(2):R37.
102. Ghufran MS, Ghosh K, Kanade SR. Aflatoxin B1 induced upregulation of protein arginine methyltransferase 5 in human cell lines. Toxicon. 2016;119: 117-21.

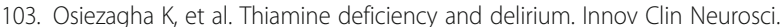
2013;10(4):26-32.

104. Starnawska A, et al. Hypomethylation of FAM63B in bipolar disorder patients. Clin Epigenetics. 2016;8:52.

105. Brucato $\mathrm{N}$, et al. Hypomethylation of the paternally inherited LRRTM1 promoter linked to schizophrenia. Am J Med Genet B Neuropsychiatr Genet. 2014;165B(7):555-63.

106. Sugawara $\mathrm{H}$, et al. Effects of quetiapine on DNA methylation in neuroblastoma cells. Prog Neuropsychopharmacol Biol Psychiatry. 2015;56: 117-21.

107. Asai T, et al. Effect of mood stabilizers on DNA methylation in human neuroblastoma cells. Int J Neuropsychopharmacol. 2013;16(10):2285-94.

108. Ito M, Roeder RG. The TRAP/SMCC/Mediator complex and thyroid hormone receptor function. Trends Endocrinol Metab. 2001;12(3):127-34.

109. Belakavadi M, Fondell JD. Role of the mediator complex in nuclear hormone receptor signaling. Rev Physiol Biochem Pharmacol. 2006;156: $23-43$

110. Ito $M$, et al. Identity between TRAP and SMCC complexes indicates novel pathways for the function of nuclear receptors and diverse mammalian activators. Mol Cell. 1999;3(3):361-70.

111. Philibert RA, et al. Population-based association analyses of the HOPA12bp polymorphism for schizophrenia and hypothyroidism. Am J Med Genet. 2001;105(1):130-4

112. Ito M, et al. The TRAP100 component of the TRAP/Mediator complex is essential in broad transcriptional events and development. EMBO J. 2002; 21(13):3464-75.

113. Ajuh $P$, et al. Functional analysis of the human CDC5L complex and identification of its components by mass spectrometry. EMBO J. 2000;19(23): 6569-81.

114. Gamo NJ, et al. Role of disrupted in schizophrenia 1 (DISC1) in stressinduced prefrontal cognitive dysfunction. Transl Psychiatry. 2013;3:e328.

115. Camargo LM, et al. Disrupted in Schizophrenia 1 Interactome: evidence for the close connectivity of risk genes and a potential synaptic basis for schizophrenia. Mol Psychiatry. 2007;12(1):74-86.

116. Karry R, Klein E, Ben Shachar D. Mitochondrial complex I subunits expression is altered in schizophrenia: a postmortem study. Biol Psychiatry. 2004;55(7): 676-84.

117. Martins-de-Souza D, et al. Proteome analysis of schizophrenia patients Wernicke's area reveals an energy metabolism dysregulation. BMC Psychiatry. 2009;9:17.

118. Hoefs SJ, et al. NDUFA10 mutations cause complex I deficiency in a patient with Leigh disease. Eur J Hum Genet. 2011;19(3):270-4.

119. Mnif L, Sellami R, Masmoudi J. Schizophrenia and Leigh syndrome, a simple comorbidity or the same etiopathogeny: about a case. Pan Afr Med J. 2015; $22 \cdot 333$

\section{Submit your next manuscript to BioMed Central and we will help you at every step:}

- We accept pre-submission inquiries

- Our selector tool helps you to find the most relevant journal

- We provide round the clock customer support

- Convenient online submission

- Thorough peer review

- Inclusion in PubMed and all major indexing services

- Maximum visibility for your research

Submit your manuscript at www.biomedcentral.com/submit 